\title{
Exponential stability of discrete-time damped Schrödinger equation
}

\section{Stabilité exponentielle de l'equation de Schrödinger amortie en temps discret}

\section{Imen Nouira and Moez Khenissi}

LAMMDA, École Supérieure des Sciences et de Technologie de Hammam Sousse, Université de Sousse, Tunisie imen.nouira5@gmail.com,moez.khenissi@essths.u-sousse.tn

ABSTRACT. This article concerns the discrete-time problem of the damped Schrödinger equation in a bounded domain and the internal stabilization problem is considered. We constructed a well-posed discrete-time scheme and we proved the exponential stability. Numerical simulations are presented to illustrate the theoretical results.

2010 Mathematics Subject Classification. 65M99, 35B65, 65M22, 35A35.

KEYWORDS. Damped Schrödinger equation, Stabilizability, Discrete-time scheme, Semi-discrete resolvent.

\section{Introduction}

Let $\Omega$ be a bounded domain of $\mathbb{R}^{d}, d \geq 1$, with smooth boundary $\partial \Omega$.

It is well known that the Schrödinger equation

$$
\left\{\begin{array}{l}
i \partial_{t} \psi-\Delta \psi=0, t \neq 0, x \in \Omega, \\
\psi_{\left.\right|_{\Omega \Omega}}=0, \\
\psi(0, x)=\psi_{0}(x), x \in \Omega,
\end{array}\right.
$$

preserves the $L^{2}$ and $H^{1}$-norms i.e. the conservation of both the mass and energy.

$$
\|\psi\|_{L^{2}(\Omega)}=\left\|\psi_{0}\right\|_{L^{2}(\Omega)}, \quad\|\nabla \psi\|_{\left(L^{2}(\Omega)\right)^{d}}=\left\|\nabla \psi_{0}\right\|_{\left(L^{2}(\Omega)\right)^{d}} .
$$

In order to obtain the $L^{2}(\Omega)$-norm decay of solutions, we add to the Schrödinger equation a dissipation term. The goal here is the stabilization problem for Schrödinger equation and it consists of acting on equation (1) by a damping term ensuring the exponential decay of $L^{2}(\Omega)$-norm of solutions as $t$ goes to infinity $[12,2]$. More precisely, the following damped Schrödinger equation is considered:

$$
\left\{\begin{array}{l}
i \partial_{t} \psi-\Delta \psi+i a(x) \psi=0, t \neq 0, x \in \Omega, \\
\psi_{\mid \partial \Omega}=0, \\
\psi(0, x)=\psi_{0}(x), \quad x \in \Omega .
\end{array}\right.
$$

Where $a(x)$ is a smooth non-negative function $\left(a \in C_{0}^{\infty}(\Omega)\right.$ ).

For any initial data $\psi_{0} \in L^{2}(\Omega)$ problem (3) has a unique solution $\psi(t, x) \in C\left(\mathbb{R}_{+}, L^{2}(\Omega)\right)$ [12]. Moreover, Liu [10] proved that the exact controllability/stabilizability of the damped wave equation implies the exact controllability/stabilizability of the damped Schrödinger equation (see Theorem 5.1. and Theorem 5.2. in [10]). Besides, Bardos et al. [6] showed, under the Geometric Control Condition 
$(\mathrm{GCC})^{1}$, the exact controllability/stabilizability of the damped wave equation. Therefore, by using the previous results of [10] and [6], we obtain the following stabilization result.

Theorem 1.1. Let $a \in C_{0}^{\infty}(\Omega)$ a positive function and $\omega=\{a(x)>0\}$ verifies the GCC. Then, there exist positive constants $\gamma$ and $C$ such that

$$
\|\psi(t)\|_{L^{2}(\Omega)} \leq C e^{-\gamma t}\left\|\psi_{0}\right\|_{L^{2}(\Omega)}, \quad \forall t>0,
$$

for every solution $\psi$ of (3) with initial data $\psi_{0} \in L^{2}(\Omega)$.

Remark 1.2. 1. Contrary to the finite speed of the waves, the Schrödinger equation has an infinite speed of propagation. Therefore, we can weaken the GCC condition used for the wave equation in the following way [2]: Every ray of geometric optics enters the region where the damping term is effective.

2. The previous exponential decay property (4) was also proved by E. Machtyngier and E. Zuazua under the Lions Condition [12].

For $\tau \in\{\Re e \tau<0\}$ the resolvent associated to problem (3) is

$$
R(\tau):=(-i \tau-\Delta+i a)^{-1},
$$

and for all $f \in L^{2}(\Omega), R(\tau) f$ is the unique solution of the following system

$$
\left\{\begin{array}{l}
(-i \tau-\Delta+i a) v=f, \text { in } \Omega \\
v_{\mid \partial \Omega}=0
\end{array}\right.
$$

It is easy to see that

$$
R(\tau) \psi_{0}=i \int_{0}^{+\infty} e^{\tau t} \psi(t) d t,
$$

with $\psi(t)$ solution of (3). It is also clear that the relation (7) defines a bounded operator from $L^{2}(\Omega)$ to $L^{2}(\Omega)$, holomorphic on $\{\Re e \tau<0\}$. Since the domain $\Omega$ is bounded then the injection $\mathcal{D}(\Delta)=$ $H_{0}^{1}(\Omega) \cap H^{2}(\Omega) \hookrightarrow L^{2}(\Omega)$ is compact. Hence, $R(\tau)$, a compact operator from $L^{2}(\Omega)$ to $L^{2}(\Omega)$, is meromorphic in $\mathbb{C}$ and has no poles in the imaginary axis.

By applying the result of Gearhart 1978, Prüss 1984 and Greiner 1985 [14] (Theorem 1.11 and Theorem 1.18 in Chapter V [8]), we have the equivalence between the exponential decay property (4) and the following resolvent property.

Proposition 1.3. Under the assumptions of Theorem 1.1. There exist positive constants $\lambda_{0}$ and $C$ such that for $\Re$ e $\tau<\lambda_{0}$ we have

$$
\|R(\tau)\|_{L^{2}(\Omega) \longrightarrow L^{2}(\Omega)} \leq C .
$$

1. Every ray of geometric optics enters the region where the damping term is effective in a uniform time. 
Let us recall that in the case of exterior trapping domain, the internal stabilization problem for Schrödinger equation was considered by Aloui and Khenissi in [2]. Moreover, under the exterior geometric control condition on the set $w=\{a(x)>0\}$, the authors proved in Theorem 1 in [2] the following resolvent estimate: There exist positive constants $c, \alpha$ and $\beta$ such that for any $\tau \in\{\tau \in$ $\mathbb{C} / \Re$ e $\tau \leq \alpha ;|\Im m \tau| \geq \beta\}$

$$
\left\|\chi R_{e x}(\tau) \chi\right\|_{L^{2}(\Omega) \longrightarrow L^{2}(\Omega)} \leq c, \quad \text { where } \chi \in C_{0}^{\infty}\left(\mathbb{R}^{d}\right)
$$

where $R_{e x}(\tau)$ is the outgoing resovent, that is to say that $R_{e x}(\tau) f=(-i \tau-\Delta+i a)^{-1} f$ is the unique finite energy solution of the following system

$$
\left\{\begin{array}{l}
(-i \tau-\Delta+i a) v=f, \text { in } \Omega, \\
v_{\mid \partial \Omega}=0 \\
v \text { outgoing. }
\end{array}\right.
$$

Notice that the case of bounded domains can be treated by the same method as in [2] with a microlocal analysis. Therefore, the resolvent estimate (8) remains true under GCC. This Geometric Control Condition is, essentially, a necessary and sufficient condition for the stabilizability of wave equations. However, due to infinite speed of propagation, this notion of "geometric control" is not completely natural in the context of the stabilizability of Schrödinger equation. In this context, Aloui et al. [4] proved the exponential decay property for Schrödinger equation with a strong damping term " $i a(x)(-\Delta)^{\frac{1}{2}} a(x) \psi^{\prime}$ without the GCC. More precisely the following equation was considered:

$$
\left\{\begin{array}{l}
i \partial_{t} \psi-\Delta \psi+i a(x)(-\Delta)^{\frac{1}{2}} a(x) \psi=f, t>0, x \in \Omega \\
\psi=0, x \in \partial \Omega \\
\psi(0, x)=\psi_{0}(x), x \in \Omega
\end{array}\right.
$$

Moreover, the authors proved the $C^{\infty}$-smoothing effect.

Problem (11) has been introduced by Aloui [1] in order to prove the following weak Kato Smoothingeffect:

$$
\|\psi\|_{L^{2}\left([\epsilon, T], H_{D}^{s+1}(\Omega)\right)} \leq C\left\|\psi_{0}\right\|_{H_{D}^{s}(\Omega)}
$$

where $0<\epsilon<T<\infty$ and $\psi$ solution of (11), thus he deduced the $C^{\infty}$-smoothing effect.

This result has been improved by Aloui et al. [3], the authors proved the Kato smoothing effect under GCC (Remarks 1.3. in [3]):

$$
\|\psi\|_{\left.L^{2}([0, T]), H^{1 / 2}(\Omega)\right)} \leq C\left\|\psi_{0}\right\|_{L^{2}(\Omega)} .
$$

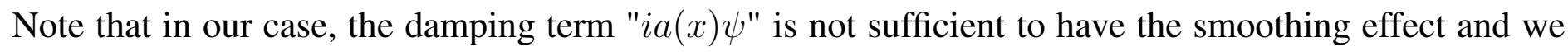
will illustrate, by numerical simulations, this result in this paper.

The main objective of this paper is to study the discrete-time problem and the numerical analysis of the damped Schrödinger equation (3). The rest of the paper is organized as follows: In section 2, We consider the Crank-Nicolson method for time discretization and we prove the well-posedness of the scheme. Then, after the construction of the semi-discrete resolvent associated to the discrete-time scheme, we have proved the exponential stability. The idea and proofs are based on appropriate estimate of the semi-discrete resolvent and the Z-Transformation. Finally, in section 3, we complete the space discretization by the finite-difference method and we present some numerical experiments. 


\section{Stabilizability: Exponential stability of the discrete-time scheme}

Throughout section 2 the dimension of the space is arbitrary i.e. we consider a bounded domain $\Omega \subset \mathbb{R}^{d}$ with $d \geq 1$. For the discretization in time $t$ of the damped Schrödinger equation (3), we consider the Crank-Nicolson method, and we obtain the following discrete-time scheme:

$$
\left\{\begin{array}{l}
i \frac{\psi^{n+1}-\psi^{n}}{\Delta t}-\Delta\left(\frac{\psi^{n+1}+\psi^{n}}{2}\right)+i a(x)\left(\frac{\psi^{n+1}+\psi^{n}}{2}\right)=0, \quad n \in \mathbb{N}, \\
\psi_{\text {| } n=0, \quad n \in \mathbb{N},}^{n+1} \\
\psi^{0} \in L^{2}(\Omega) .
\end{array}\right.
$$

Where we use the following notations:

1. $t_{n}: n$-th time step determined as: $t_{0}=0, t_{n}=n \Delta_{t}, n \in \mathbb{N}, \Delta_{t}>0$ is the time step.

2. $\psi^{n}($.$) : approximation of \psi\left(t_{n},.\right)$.

Proposition 2.1. The discrete-time scheme (12) verifies the following properties:

1. The scheme (12) is well posed in $L^{2}(\Omega)$.

2. The scheme (12) is stable, and for all $\psi^{0} \in L^{2}(\Omega)$ we have:

$$
\forall n \in \mathbb{N}^{*}, \quad\left\|\psi^{n}\right\|_{L^{2}(\Omega)} \leq\left\|\psi^{0}\right\|_{L^{2}(\Omega)} .
$$

3. The scheme (12) is convergent.

4. If $a=0$ then the discrete-time scheme without damping term is a conservative scheme and we have:

$$
\forall n \in \mathbb{N}, \quad\left\|\psi^{n+1}\right\|_{L^{2}(\Omega)}=\left\|\psi^{n}\right\|_{L^{2}(\Omega)} .
$$

Proof. We write the scheme (12) in the following form:

$$
\left(\frac{2 i}{\Delta_{t}}-\Delta+i a\right) \psi^{n+1}=F_{n}, \text { where } F_{n}=\left(\frac{2 i}{\Delta_{t}}+\Delta-i a\right) \psi^{n} .
$$

As indicated in the Introduction, $R(\tau)=(-i \tau-\Delta+i a)^{-1}$ is the resolvent of problem (3) holomorphic on $\{\Re e \tau<0\}$. Besides, we have $\Re e\left(-\frac{2}{\Delta_{t}}\right)=-\frac{2}{\Delta_{t}}<0$ so by using proposition 1.3 we get: $R\left(-\frac{2}{\Delta_{t}}\right)=$ $\left(\frac{2 i}{\Delta_{t}}-\Delta+i a\right)^{-1}$ is bounded. Hence,

$$
\psi^{n+1}=R\left(-\frac{2}{\triangle_{t}}\right) F_{n} .
$$

Denote by $A=-\Delta+i a, A$ is defined from $\mathcal{D}(A)$ to $L^{2}(\Omega)$ where $\mathcal{D}(A)=H_{0}^{1}(\Omega) \cap H^{2}(\Omega)$. We start by proving the well-posedness of scheme (12) in $\mathcal{D}(A)$ then we conclude the well posedness in $L^{2}(\Omega)$ by density. Let $\psi^{0} \in \mathcal{D}(A)$, So we have

$$
\left\{\begin{array}{l}
\psi^{n+1}=R\left(-\frac{2}{\Delta_{t}}\right) F_{n}, n \in \mathbb{N} \\
\psi^{0} \in \mathcal{D}(A)
\end{array}\right.
$$


Now, we prove by recurrence that the scheme (12) has a solution $\psi^{n} \in \mathcal{D}(A)$. Suppose that $\psi^{n} \in \mathcal{D}(A)$ then $F_{n} \in L^{2}(\Omega)$, and according to the fact that the resolvent $R(\tau)$ is defined for $\{\Re e \tau<0\}$ as an operator from $L^{2}(\Omega)$ to $\mathcal{D}(A)$, then equation (15) has unique solution $\psi^{n+1} \in \mathcal{D}(A)$. Therefore, we conclude the well-posedness of scheme (12) in $L^{2}(\Omega)$ by density.

Taking into account that $C_{0}^{\infty}(\Omega)$ is dense in $L^{2}(\Omega)$, in this proof it is sufficient to show that the stability estimate (13) holds for all $\psi^{0} \in C_{0}^{\infty}(\Omega)$, therefore we conclude proposition 2.1 by density. Denote by $\|\cdot\|_{2}$ the norm in $L^{2}(\Omega)$.

Let $n \in \mathbb{N}^{*}$ and $k \in \mathbb{N}$, we multiply (12) by $\left(\overline{\psi^{k+1}+\psi^{k}}\right)$ and integrate over $\Omega$, we get:

$$
\int_{\Omega}\left[i \frac{\psi^{k+1}-\psi^{k}}{\Delta_{t}}-\Delta\left(\frac{\psi^{k+1}+\psi^{k}}{2}\right)+i a(x)\left(\frac{\psi^{k+1}+\psi^{k}}{2}\right)\right]\left(\overline{\psi^{k+1}+\psi^{k}}\right) d x=0 .
$$

Which yields to:

$$
\begin{gathered}
\frac{i}{\triangle_{t}}\left(\int_{\Omega}\left|\psi^{k+1}\right|^{2}-\left|\psi^{k}\right|^{2}+\psi^{k+1} \overline{\psi^{k}}-\psi^{k} \overline{\psi^{k+1}} d x\right)+\frac{1}{2}\left\|\nabla\left(\psi^{k+1}+\psi^{k}\right)\right\|_{2}^{2} \\
+\frac{i}{2}\left\|\sqrt{a(x)}\left(\psi^{k+1}+\psi^{k}\right)\right\|_{2}^{2}=0,
\end{gathered}
$$

and since

$$
\psi^{k+1} \overline{\psi^{k}}-\psi^{k} \overline{\psi^{k+1}}=\psi^{k+1} \overline{\psi^{k}}-\overline{\psi^{k+1} \overline{\psi^{k}}}=2 i \Im m\left(\psi^{k+1} \overline{\psi^{k}}\right),
$$

then it follows that:

$$
\begin{gathered}
\frac{i}{\triangle_{t}}\left(\left\|\psi^{k+1}\right\|_{2}^{2}-\left\|\psi^{k}\right\|_{2}^{2}+2 i \int_{\Omega} \Im m\left(\psi^{k+1} \overline{\psi^{k}}\right) d x\right)+\frac{1}{2}\left\|\nabla\left(\psi^{k+1}+\psi^{k}\right)\right\|_{2}^{2} \\
+\frac{i}{2}\left\|\sqrt{a(x)}\left(\psi^{k+1}+\psi^{k}\right)\right\|_{2}^{2}=0 .
\end{gathered}
$$

Considering the imaginary part of the last equation, we get:

$$
\frac{1}{\triangle_{t}}\left(\left\|\psi^{k+1}\right\|_{2}^{2}-\left\|\psi^{k}\right\|_{2}^{2}\right)+\frac{1}{2}\left\|\sqrt{a(x)}\left(\psi^{k+1}+\psi^{k}\right)\right\|_{2}^{2}=0 .
$$

Then

$$
\frac{1}{\triangle_{t}}\left(\left\|\psi^{k+1}\right\|_{2}^{2}-\left\|\psi^{k}\right\|_{2}^{2}\right)=-\frac{1}{2}\left\|\sqrt{a(x)}\left(\psi^{k+1}+\psi^{k}\right)\right\|_{2}^{2} \leq 0 .
$$

Thus,

$$
\left\|\psi^{k+1}\right\|_{2}-\left\|\psi^{k}\right\|_{2} \leq 0 .
$$

Hence,

$$
\sum_{k=0}^{n-1}\left(\left\|\psi^{k+1}\right\|_{2}-\left\|\psi^{k}\right\|_{2}\right) \leq 0
$$


Which implies that for all $n$ in $\mathbb{N}^{*}$,

$$
\left\|\psi^{n}\right\|_{2} \leq\left\|\psi^{0}\right\|_{2}
$$

which achieves the proof of (13).

Thanks to the fact that the Crank-Nicolson time discretization scheme is a consistant scheme for the linear Schrödinger equation. Therefore, by using the Lax-Richtmyer Equivalence Theorem (Theorem 1 in [16]), consistency and stability imply the convergence of scheme 12.

When $a=0$, the Crank-Nicolson scheme verifies the mass conservation law: In fact, by equality (16) from this proof, we obtain: $\forall n \in \mathbb{N},\left\|\psi^{n+1}\right\|_{L^{2}(\Omega)}=\left\|\psi^{n}\right\|_{L^{2}(\Omega)}$.

The main result of this paper is to prove the following exponential stability of the discrete-time damped Schrödinger equation.

Theorem 2.2. Let us suppose that $w=\{a(x)>0\}$ verifies the GCC. Then, there exist positive constants $c, \gamma$ such that

$$
\forall n \in \mathbb{N}^{*}, \quad\left\|\psi^{n}\right\|_{L^{2}(\Omega)} \leq c e^{-\gamma t_{n}}\left\|\psi^{0}\right\|_{L^{2}(\Omega)},
$$

where $\psi^{n}$ denotes the solution of (12).

\subsection{Proof of Theorem 2.2}

The proof of Theorems 2.2 is based on appropriate semi-discrete resolvent estimate. We first define the semi-discrete resolvent associated to discrete-time scheme (12), therefore we use the Z-transformation which is the discrete analogue of the Laplace-transformation.

We consider a bounded function $\psi: \mathbb{R}_{+} \rightarrow L^{2}(\Omega)$. The Laplace transform of $\psi$ is

$$
L(\psi)(\tau)=\int_{0}^{+\infty} \psi(t) e^{t \tau} d t, \tau=\lambda+i \sigma \in \mathbb{C}
$$

And the Z-transformation of $\left\{\psi^{m}\right\}$ is (Definition 3.1 in Appendix):

$$
Z\left\{\psi^{m}\right\}:=\sum_{m=0}^{+\infty} \psi^{m} z^{-m}, z \in \mathbb{C},|z|>r, r \text { is the radius of convergence. }
$$

To obtain a quadrature approach of the Laplace transform (18) we take a particular case of Z-transform with $z=e^{-\Delta_{t} \tau}, \psi^{m}=\psi\left(t_{m}\right)$ and multiply the series (19) by $\triangle_{t}$ :

$$
\widehat{\psi}(\tau)=\Delta_{t} \sum_{m \in \mathbb{N}} \psi^{m} e^{m \Delta_{t} \tau}, \tau \in \mathbb{C}, \tau=\lambda+i \sigma
$$

The condition $\lambda<0$ is sufficient for the convergence of the series (20). In fact We know that the sequence $\left\{\psi^{m}\right\}_{m \in \mathbb{N}}$ is bounded, then there exists a positive constant $C$ such that

$$
\left\|\psi^{m} e^{m \Delta_{t} \tau}\right\|_{L^{2}(\Omega)}=\left\|\psi^{m}\right\|_{L^{2}(\Omega)} e^{m \Delta_{t} \lambda} \leq C e^{m \Delta_{t} \lambda}, m \in \mathbb{N} .
$$

Moreover, the geometric series $\sum_{m \geq 0} e^{m \Delta_{t} \lambda}$ converges if and only if $\left|e^{\Delta_{t} \lambda}\right|<1$ i.e. $\lambda<0$. 
In our particular case, $\widehat{\psi}$ is $\frac{2 \pi}{\Delta_{t}}$-periodic with respect to $\sigma$ (the imaginary part of $\tau$ ), then we shall take $\left[-\frac{\pi}{\triangle_{t}}, \frac{\pi}{\triangle_{t}}\right]$ as the fundamental domain of $\sigma$. Therefore, we define the Z-transform of $\left\{\psi^{m}\right\}$ as follows:

$$
Z\left\{\psi^{m}\right\}:=\widehat{\psi}(\tau)=\triangle_{t} \sum_{m \in \mathbb{N}} \psi^{m} e^{m \triangle_{t} \tau}, \tau=\lambda+i \sigma, \text { with } \lambda<0 \text { and } \sigma \in\left[-\frac{\pi}{\triangle_{t}}, \frac{\pi}{\triangle_{t}}\right] .
$$

Let $z=e^{-\Delta_{t} \tau}$, remark that $Z\left\{\psi^{m+1} \pm \psi^{m}\right\}=(z \pm 1) \widehat{\psi}-z \Delta_{t} \psi^{0}$ and apply Z-Transform (21) on scheme (12), we obtain

$$
\frac{i}{\triangle_{t}}\left((z-1) \widehat{\psi}-z \Delta_{t} \psi^{0}\right)-\frac{1}{2} \Delta\left((z+1) \widehat{\psi}-z \Delta_{t} \psi^{0}\right)+\frac{i}{2} a(x)\left((z+1) \widehat{\psi}-z \Delta_{t} \psi^{0}\right)=0 .
$$

Therefore,

$$
\left(\frac{2 i(z-1)}{\Delta_{t}(z+1)}-\Delta+i a\right) \widehat{\psi}=\frac{z}{z+1}\left(2 i \psi^{0}+\Delta_{t}(-\Delta+i a) \psi^{0}\right) .
$$

Now we use the following Remark.

Remark 2.3. Knowing that $\Omega$ is a bounded domain and $(-\Delta+i a)^{-1}$ is a compact operator from $L^{2}(\Omega)$ to $L^{2}(\Omega)$, therefore the eigenfunctions $\left\{\varphi_{k}\right\}_{k}$ form a Riesz basis for $L^{2}(\Omega)$ [17] and the spectrum is formed by discrete eigenvalues $\left\{\nu_{k}\right\}_{k}$. The eigenfunction $\varphi_{k}$ is defined by

$$
\left\{\begin{array}{l}
\left(-\Delta_{D}+i a\right) \varphi_{k}=\nu_{k} \varphi_{k}, x \in \Omega \\
\varphi_{k}=0, x \in \partial \Omega
\end{array}\right.
$$

with $\nu_{k}$ being the eigenvalue associated to $\varphi_{k}$.

Let $\psi^{0} \in \mathcal{D}(A)$ with $\mathcal{D}(A):=H_{0}^{1}(\Omega) \cap H^{2}(\Omega)$ being the domain of the operator $A:=-\Delta+i a$. Taking into account that $\mathcal{D}(A)$ is dense in $L^{2}(\Omega)$, in the rest of this paper it is sufficient to prove some results in $\mathcal{D}(A)$ hence we conclude by density in $L^{2}(\Omega)$.

Remark 2.3 implies that the initial data $\psi^{0}$ can be written as a linear combination of the basis $\left\{\varphi_{k}\right\}_{k}$. There exist $\beta_{k} \in \mathbb{C}$ such that

$$
\psi^{0}=\sum_{k} \beta_{k} \varphi_{k}<\infty
$$

and

$$
-\Delta \psi^{0}+i a \psi^{0}=\sum_{k} \beta_{k}(-\Delta+i a) \varphi_{k}=\sum_{k} \beta_{k} \nu_{k} \varphi_{k}<\infty
$$

Hence, Substituting $z=e^{-\Delta_{t} \tau}$ in equality (23) yields to:

$$
\left(-\frac{2 i\left(1-e^{-\Delta_{t} \tau}\right)}{\Delta_{t}\left(1+e^{-\Delta_{t} \tau}\right)}-\Delta+i a\right) \widehat{\psi}=\frac{2 i}{1+e^{\Delta_{t} \tau}} \sum_{k} \beta_{k} \varphi_{k}+\frac{\Delta_{t}}{1+e^{\Delta_{t} \tau}} \sum_{k} \nu_{k} \beta_{k} \varphi_{k} .
$$

Therefore we obtain

$$
\widehat{\psi}(\tau)=R_{\Delta_{t}}(\tau)\left(\frac{2 i}{1+e^{\Delta_{t} \tau}} \sum_{k} \beta_{k} \varphi_{k}+\frac{\Delta_{t}}{1+e^{\Delta_{t} \tau}} \sum_{k} \nu_{k} \beta_{k} \varphi_{k}\right)
$$


where

$$
R_{\triangle_{t}}(\tau)=\left(-i \alpha_{\Delta_{t}}(\tau)-\Delta+i a\right)^{-1}, \text { with } \alpha_{\Delta_{t}}(\tau)=\frac{2\left(1-e^{-\tau \Delta_{t}}\right)}{\triangle_{t}\left(1+e^{-\tau \Delta_{t}}\right)} .
$$

Finally, we conclude the following definition.

Definition 2.4. The semi-discrete resolvent associated to the Crank-Nicolson scheme (12) is:

$$
R_{\Delta_{t}}(\tau)=\left(-i \alpha_{\Delta_{t}}(\tau)-\Delta+i a\right)^{-1}, \text { with } \alpha_{\Delta_{t}}(\tau)=\frac{2\left(1-e^{-\tau \Delta_{t}}\right)}{\triangle_{t}\left(1+e^{-\tau \Delta_{t}}\right)}
$$

To prove Theorem 2.2 we will use in particular Proposition 2.5 and Corollary 2.6 below.

Proposition 2.5. Let us suppose that $w=\{a(x)>0\}$ verifies the GCC. Then, there exists $\eta>0$ such that for all $\Delta_{t}<\eta$, there exist positive constants $\lambda_{0}$ and $C$ such that for $|\Re e \tau|<\lambda_{0}$ and $\varphi \in L^{2}(\Omega)$ we have

$$
\left\|R_{\Delta_{t}}(\tau) \varphi\right\|_{L^{2}(\Omega)} \leqslant c\|\varphi\|_{L^{2}(\Omega)}
$$

Proof. Knowing that $\tau=\lambda+i \sigma$, we remark that:

$$
R_{\triangle_{t}}(\tau)=R\left(\alpha_{\Delta_{t}}\right)
$$

where $R$ is the resolvent of the damped Schrödinger equation defined by (5) and $R_{\triangle_{t}}$ is the semi-discrete resolvent of the Crank-Nicolson scheme defined by (29).

By using proposition 1.3 we have, under GCC on the set $w=\{a(x)>0\}$, there exist positive constants $\lambda_{0}$ and $C$ such that for $\left|\Re e \alpha_{\Delta_{t}}\right|<\lambda_{0}$ and $\varphi \in L^{2}(\Omega)$

$$
\left\|R\left(\alpha_{\Delta_{t}}\right) \varphi\right\|_{L^{2}(\Omega)} \leq C\|\varphi\|_{L^{2}(\Omega)}
$$

Calculating the limit of $\alpha_{\Delta_{t}}(\tau)$ when $\Delta_{t}$ tends to 0 , we find

$$
\lim _{\Delta_{t} \longrightarrow 0} \alpha_{\Delta_{t}}(\tau)=\tau \text {. }
$$

By using the above limit, we can conclude Proposition 2.5 by identification from proposition 1.3. In fact, by using (32) we obtain

$$
\lim _{\Delta_{t} \rightarrow 0} \Re e\left(\alpha_{\Delta_{t}}(\tau)\right)=\Re e(\tau)=\lambda \quad \text { and } \quad \lim _{\Delta_{t} \rightarrow 0} \Im m\left(\alpha_{\Delta_{t}}(\tau)\right)=\Im m(\tau)=\sigma .
$$

Then we get (when $\Delta_{t} \rightarrow 0$ and $\left.(\lambda, \sigma) \neq(0,0)\right)$

$$
\Re e\left(\alpha_{\Delta_{t}}(\tau)\right) \sim \lambda \text { and } \Im m\left(\alpha_{\Delta_{t}}(\tau)\right) \sim \sigma .
$$

Hence, there exist positive constants $c_{1}, c_{2}, \eta$ such that for all $\triangle_{t}<\eta$,

$$
c_{1}|\lambda|<\left|\Re e\left(\alpha_{\Delta_{t}}(\tau)\right)\right|<c_{2}|\lambda|
$$

Therefore, by identification using proposition 1.3 i.e. using all its hypotheses and conditions, we conclude Proposition2.5.

Corollary 2.6. Under the assumptions of Proposition 2.5. There exists a positive constant c such that

$$
\|\widehat{\psi}(\tau)\|_{L^{2}(\Omega)} \leq c\left\|\psi^{0}\right\|_{L^{2}(\Omega)},
$$

where $\widehat{\psi}(\tau)$ is the Z-transform of $\left\{\psi^{n}\right\}$ and $\psi^{n}$ denotes the solution of Crank-Nicolson scheme(12). 
Proof. Taking into account that $\mathcal{D}(A)=H_{0}^{1}(\Omega) \cap H^{2}(\Omega)$ is dense in $L^{2}(\Omega)$, it is sufficient to prove (33) for initial data in $\mathcal{D}(A)$. From equation $(27)$ we have

$$
\widehat{\psi}(\tau)=R_{\Delta_{t}}(\tau)\left(\frac{2 i}{1+e^{\Delta t} \tau} \sum_{k} \beta_{k} \varphi_{k}+\frac{\Delta_{t}}{1+e^{{ }_{t} \tau}} \sum_{k} \nu_{k} \beta_{k} \varphi_{k}\right)
$$

and since,

$$
\begin{aligned}
\lim _{\Delta t \rightarrow 0} & \frac{2 i}{1+e^{\Delta_{t} \tau}} \sum_{k} \beta_{k} \varphi_{k}+\frac{\triangle_{t}}{1+e^{\Delta_{t} \tau}} \sum_{k} \nu_{k} \beta_{k} \varphi_{k}=i \sum_{k} \beta_{k} \varphi_{k}=i \psi^{0}, \text { i.e. } \\
& \frac{2 i}{1+e^{\Delta_{t} \tau}} \sum_{k} \beta_{k} \varphi_{k}+\frac{\triangle_{t}}{1+e^{\Delta_{t} \tau}} \sum_{k} \nu_{k} \beta_{k} \varphi_{k} \underset{\triangle_{t} \rightarrow 0}{\sim} i \psi^{0} .
\end{aligned}
$$

Thus, there exist positive constants $c_{1}, c_{2}, \eta$ such that for all $\triangle_{t}<\eta$,

$$
c_{1}\left|i \psi^{0}\right|<\left|\frac{2 i}{1+e^{\Delta_{t} \tau}} \sum_{k} \beta_{k} \varphi_{k}+\frac{\triangle_{t}}{1+e^{\Delta_{t} \tau}} \sum_{k} \nu_{k} \beta_{k} \varphi_{k}\right|<c_{2}\left|i \psi^{0}\right|,
$$

therefore, from Proposition 2.5 and under the same assumptions, there exists $C_{1}, C_{2}>0$ such that

$$
\begin{aligned}
& \left\|R_{\Delta_{t}}(\tau)\left(\frac{2 i}{1+e^{\Delta_{t} \tau}} \sum_{k} \beta_{k} \varphi_{k}+\frac{\Delta_{t}}{1+e^{\Delta_{t} \tau}} \sum_{k} \nu_{k} \beta_{k} \varphi_{k}\right)\right\|_{L^{2}(\Omega)} \\
& \leqslant C_{1}\left\|\frac{2 i}{1+e^{\Delta_{t} \tau}} \sum_{k} \beta_{k} \varphi_{k}+\frac{\triangle_{t}}{1+e^{\Delta_{t} \tau}} \sum_{k} \nu_{k} \beta_{k} \varphi_{k}\right\|_{L^{2}(\Omega)} \leqslant C_{2}\left\|\psi^{0}\right\|_{L^{2}(\Omega)},
\end{aligned}
$$

which achieves the proof of Corollary 2.6.

Now we tackle the proof of Theorem 2.2.

We denote by $\|$. $\|_{2}$ the norm in $L^{2}(\Omega)$. By defining the inverse Z-transformation (Theorem 3.3 in Appendix) we have:

$$
\psi^{n}=Z^{-1}\left\{Z\left\{\psi^{n}\right\}\right\}=\frac{1}{2 \pi i} \oint_{C} Z\left\{\psi^{n}\right\} z^{n-1} d z, \quad n \in \mathbb{N} .
$$

By changing the variable $z=e^{-\tau \Delta t}, \tau=\lambda+i \sigma$, and since we integrate on a circle around the origin then $\sigma \in\left[-\frac{\pi}{\Delta_{t}}, \frac{\pi}{\Delta_{t}}\right]$, and we obtain

$$
\psi^{n}=\frac{-\Delta_{t}}{2 i \pi} \int_{\lambda_{0}-i \frac{\pi}{\Delta_{t}}}^{\lambda_{0}+i \frac{\pi}{\Delta_{t}}} \widehat{\psi}(\tau) e^{-\tau t_{n}} d \tau
$$

where $\widehat{\psi}(\tau)$ is the Z-transform of $\left\{\psi^{n}\right\} \cdot \tau=\lambda_{0}+i \sigma$, hence

$$
\psi^{n}=\frac{-\Delta_{t}}{2 i \pi} e^{-\lambda_{0} t_{n}} \int_{-\frac{\pi}{\Delta_{t}}}^{\frac{\pi}{\Delta_{t}}} \widehat{\psi}(\tau) e^{-i \sigma t_{n}} d \sigma
$$


By using estimate (33) of Corollary 2.6, under the same assumptions we have:

$$
\|\widehat{\psi}(\tau)\|_{2} \leq c\left\|\psi^{0}\right\|_{2}
$$

Then, by using (35) and under the assumptions of Proposition 2.5, we get $c>0$ such that

$$
\left\|\psi^{n}\right\|_{2} \leq \frac{\Delta_{t}}{2 \pi} e^{-\lambda_{0} t_{n}} \int_{-\frac{\pi}{\Delta t}}^{\frac{\pi}{\Delta t}} c\left\|\psi^{0}\right\|_{2} d \sigma=c e^{-\lambda_{0} t_{n}}\left\|\psi^{0}\right\|_{2} .
$$

Which implies the exponential stability (17).

\section{Numerical simulations}

In the numerical level, we will discuss some experiments that illustrate the results and the theory of this work. Therefore, we consider the dimension of the space is one and $\Omega=]-L, L[, L>0$. The spatial discretization will be, for simplicity, the finite-difference method. The full discretized Crank-Nicolson Finite-difference scheme is designated by CNFD and is given as follows:

$$
\left\{\begin{array}{l}
\mathrm{i} \frac{\psi_{j}^{n+1}-\psi_{j}^{n}}{\Delta_{t}}-\Delta_{h}\left(\frac{\psi_{j}^{n+1}+\psi_{j}^{n}}{2}\right)+\mathrm{i} a_{j}\left(\frac{\psi_{j}^{n+1}+\psi_{j}^{n}}{2}\right)=0,1 \leqslant j \leqslant J-1, n \in \mathbb{N} \\
\psi_{0}^{n+1}=\psi_{J}^{n+1}=0, \quad n \in \mathbb{N} \\
\psi_{j}^{0}, \quad 1 \leqslant j \leqslant J-1 .
\end{array}\right.
$$

Here we define:

1. $x_{j}: j$-th net point on $(-L, L)$ determined as: $x_{0}=-L, x_{j}=x_{j-1}+h, 1 \leqslant j \leqslant J-1, x_{J}=$ $L, D=2 L$ dimension of the domain, $h=\frac{2 L}{J}$ is the space step, $J$ is the number of space steps.

2. $\psi_{j}($.$) : approximation of \psi\left(., x_{j}\right)$ and $\psi_{j}^{n}$ : approximation of $\psi\left(t_{n}, x_{j}\right)$.

3. $a_{j}=a\left(x_{j}\right)$ and $a^{h}=\left\{a_{j}\right\}$.

The discrete operator $\Delta_{h}$ is the classical second-order finite-difference approximation of $\partial_{x}^{2}$ :

$$
\Delta_{h} \psi_{j}=\frac{\psi_{j+1}-2 \psi_{j}+\psi_{j-1}}{h^{2}}
$$

We associate the matrix $\left[\Delta_{h}\right] \in \mathcal{M}_{J-1}(\mathbb{C})$ to the discrete operator $\Delta_{h}$. Consequently, the finite-difference spacial discretization leads to the following linear algebraic equation:

$$
\left\{\begin{array}{l}
A^{n+1} \psi^{n+1, h}=b^{n}, \quad n \in \mathbb{N}, \\
\psi^{0} .
\end{array}\right.
$$

Where

$$
A^{n+1}=\frac{i}{\triangle_{t}}[I d]-\frac{1}{2}\left[\Delta_{h}\right]+\frac{i}{2}\left[a^{h}\right] \quad \text { and } \quad b^{n}=\left(\frac{i}{\triangle_{t}}[I d]+\frac{1}{2}\left[\Delta_{h}\right]-\frac{i}{2}\left[a^{h}\right]\right) \psi^{n, h},
$$

with $\psi^{n, h}=\left(\begin{array}{c}\psi_{1}^{n} \\ \psi_{2}^{n} \\ \vdots \\ \psi_{J-1}^{n}\end{array}\right),[I d]$ is the identity matrix in $\mathcal{M}_{J-1}(\mathbb{C})$, and $\left[a^{h}\right]=\left(\begin{array}{c}a_{1} \\ a_{2} \\ \vdots \\ a_{J-1}\end{array}\right)$. 
If we use another type of time discretization as the Implicit-Euler method. One can utilize the mass conservation law of the linear Schrödinger equation to compare the two schemes. The Implicit Euler scheme with a finite-difference approximation is designated by IEFD. In the first experiment, We use a time step equal to $\triangle_{t}=10^{-3}$ for a final computational time $T=4$ and we consider $a=0$. Figures $1 \mathrm{a}$ and $1 \mathrm{~b}$ show the evolution of the $L^{2}$-norm for these two schemes CNFD and IEFD. Figure 1a illustrates the mass conservation law of the CNFD scheme as we have checked in (14) in Proposition 2.1. In contrast, Figure $1 \mathrm{~b}$ shows that this property is not satisfied by IEFD scheme. Which implies the reason of our choice of Crank-Nicolson method.

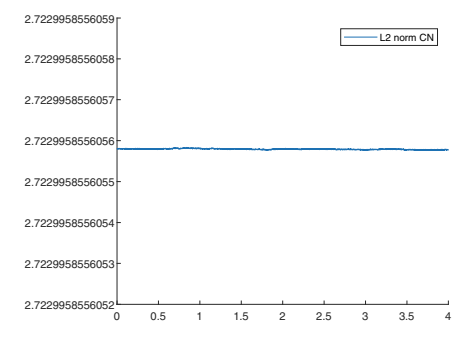

(a) Evolution of the $L^{2}$-norm for CNFD

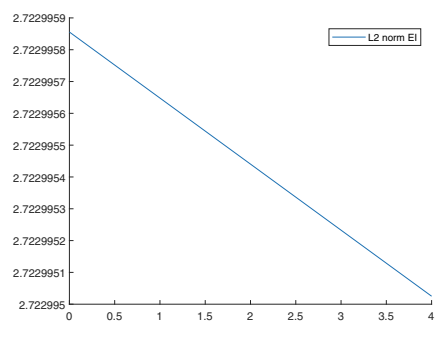

(b) Evolution of the $L^{2}$-norm for IEFD

Figure 1. Evolution of the $L^{2}$-norm for CNFD and IEFD.

Finally, in the numerical simulations, we consider the Crank-Nicolson Finite-Difference (CNFD) scheme (36) combined with GMRES method to solve the linear algebraic equation (38). We use a time step equal to $\triangle_{t}=10^{-3}$ for a final computational time $T=2\left(T:=N \delta_{t}, N>0\right)$. The finite-difference discretization scheme considers $J=2^{7}$ points for the computational domain $\left.\Omega=\right]-40,40[$.

Figure 2 displays the exponential stability (Theorem 2.2). Here we consider a non-zero damping function $a(x)$, we choose it as a large Gaussian. Besides, we choose a smooth initial data $\psi_{0}$ as a Gaussian function. The damping function $a(x)$ and the initial data $\psi_{0}$ are drawn in Figure 2a. Now, we can show numerically the exponential decay of the $L^{2}$-norm of the discrete solution by plotting $\ln \left(\frac{\left\|\psi^{N}\right\|_{2}}{\left\|\psi^{0}\right\|_{2}}\right)$ and obtaining a graph of line: $y=p t+d$, with $p<0$, which is delineated by Figure $2 \mathrm{~b}$. In fact, by using Theorem 2.2, we have positive constants $\gamma, c$ such that, $\forall N \in \mathbb{N}^{*},\left\|\psi^{N}\right\|_{L^{2}} \leq c e^{-\gamma t_{N}}\left\|\psi^{0}\right\|_{L^{2}}$, thus applying the function $\ln ($.$) we get \ln \left(\frac{\left\|\psi^{N}\right\|_{2}}{\left\|\psi^{0}\right\|_{2}}\right) \leq \ln (c)-\gamma t_{N}$. Figure $2 \mathrm{c}$ shows the evolution of the solution obtained by the CNFD scheme when $t \in(0,2)$ which demonstrates the dissipation effect of the damping term.

Figures 3 and 4 aim to consider the damped Schrödinger equation with a non-smooth initial data. Therefore we choose $\psi_{0}=\chi_{\omega}, \omega \subset \Omega$ is a characteristic function. We also choose the damping function $a(x)$ as a Gaussian function in Figure 3, and as a stronger Gaussian in Figure 4. The damping function $a(x)$ covers all the singularities in order to eliminate the edge effects of the non-smooth initial data. Figures $3 \mathrm{a}$ and $4 \mathrm{a}$ show the non-smooth initial data $\psi_{0}$ and the damping function $a(x)$. We report in Figures $3 b, 3 c, 3 d, 4 b$ and $4 c$ the moduli of the numerical solution at different times until $T=2$ for the time step $\triangle_{t}=10^{-3}$. We end the analysis by showing the evolution of the solution when $t \in(0,2)($ see Figures $3 e$ and $4 d)$. 


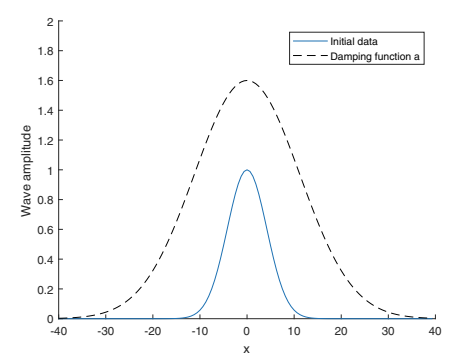

(a) Initial data $\psi_{0}$ and Damping function $a(x)$

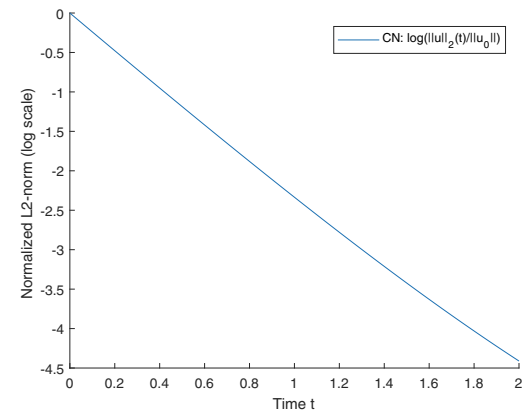

(b) Exponential stability.

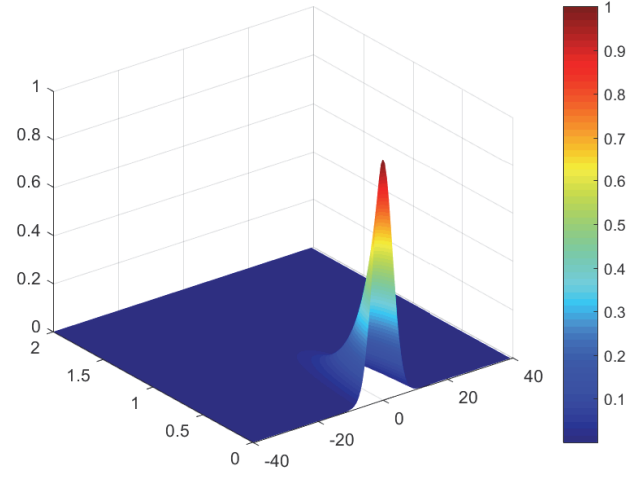

(c) Evolution of the numerical solution when $t \in(0,2)$.

Figure 2. The exponential stability and the the dissipation effect.

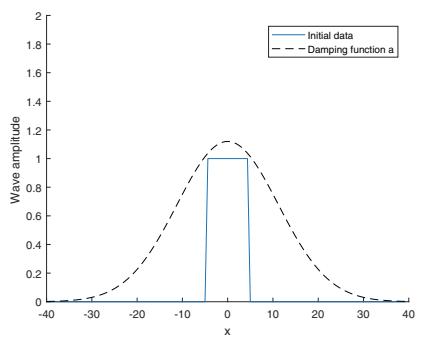

(a) Non-smooth initial data and $a(x)$ is a Gaussian $\left(\|a\|_{\infty}=\right.$ $1.1)$

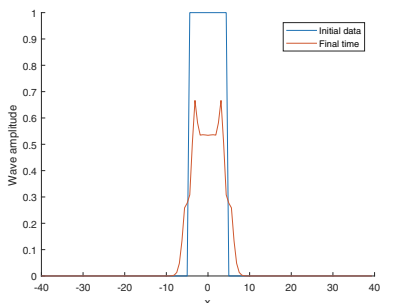

(b) Initial data and the solution at $T=0.5$

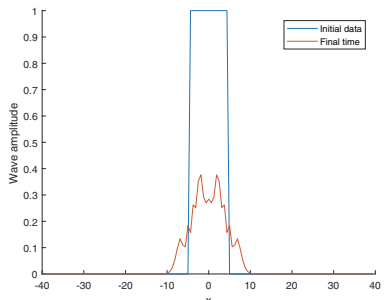

(c) Initial data and the solution at $T=1$

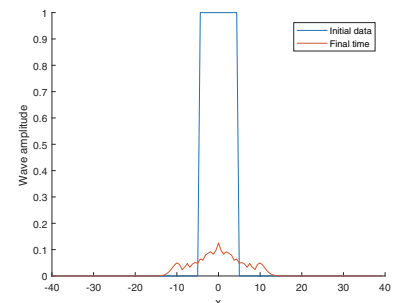

(d) Initial data and the solution at $T=2$

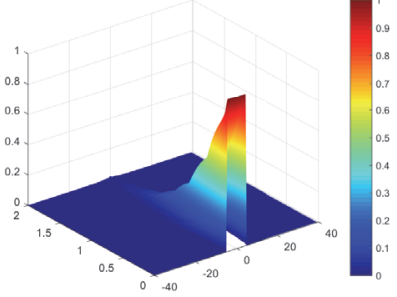

(e) Evolution of the solution when $t \in(0,2)$

Figure 3. Evolution of the numerical solution respectively at $\mathrm{T}=0.5,1,2$ and $t \in[0,2]$.

Figures 3 and 4 show that there is a propagation of singularities of solution and the solution remains singular. Which illustrate that the damping term " $i a(x) \psi$ " is not sufficient to have the smoothing effect. Moreover, Figure 4 demonstrates that, by using a stronger damping function $a(x)$, the dissipation is faster.

Figures 3 and 4 showed that the damped Schrödinger equation does not verify the smoothing effect, which leaves open the question of whether it is possible to find a way to obtain the smoothing effect. There are many ideas and isues related to the smoothing of the Schrödinger equation. For example, many authors use the method of regularization by adding to the equation a strong dissipative term. In particular, as mentioned in the Introduction, we cite the work of Aloui [1] in which he introduced the Schrödinger

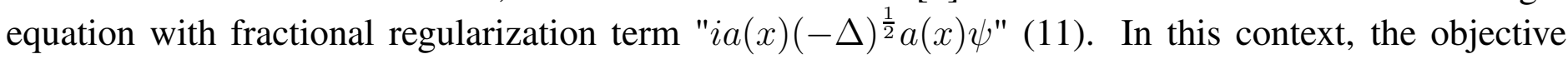
of our work in [13] is to prove the smoothing effect of the discrete-time scheme for the regularized Schrödinger equation (11). Therefore in [13], we have showed theoretically and numerically the smooth- 
ing effect of the discrete-time regularized Schrödinger equation. Moreover, in order to compare the case with damping term " $i a(x) \psi$ " and the case with fractional regularization term " $i a(x)(-\Delta)^{\frac{1}{2}} a(x) \psi$ " we cite some numerical results from [13] in Figure 5 in which the singularities disappear and the solution is smooth which is not the case in Figures 3 and 4. This comparison also illustrates that the damping term "ia $(x) \psi$ " is not sufficient to have the smoothing effect.

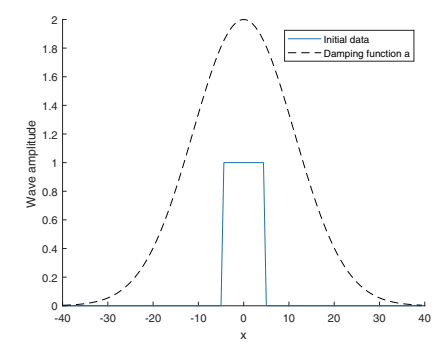

(a) Non-smooth initial data and $a(x)$ is a stronger Gaussian $\left(\|a\|_{\infty}=2\right)$.

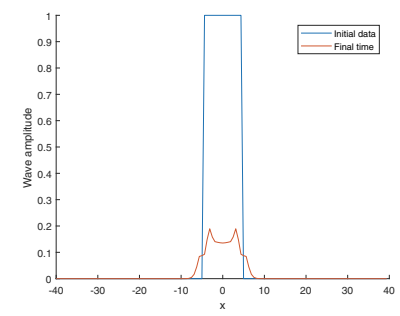

(b) Initial data and the solution at $T=0.5$

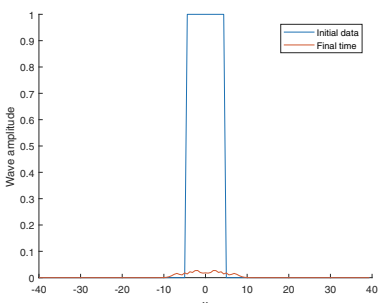

(c) Initial data and the solution at $T=1$

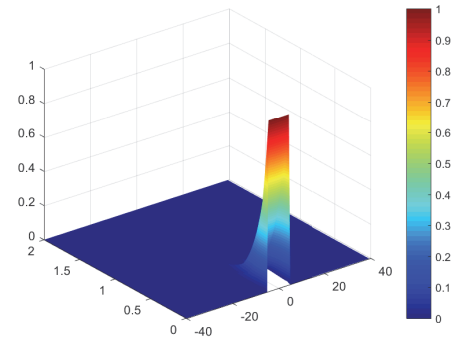

(d) Evolution of the solution when $t \in(0,2)$

Figure 4. Evolution of the numerical solution respectively at $\mathrm{T}=0.5,1$ and $t \in[0,2]$.

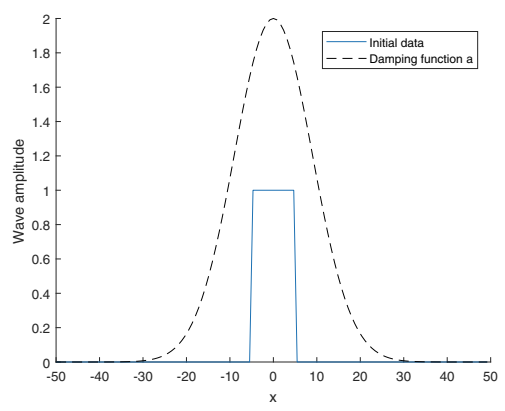

(a) Initial data and $a(x)$ is a Gaussian.

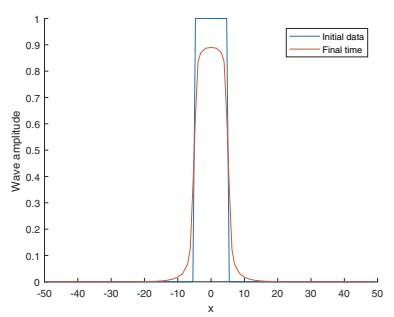

(b) $\psi_{0}$ and numerical solution at $T=0.2$.

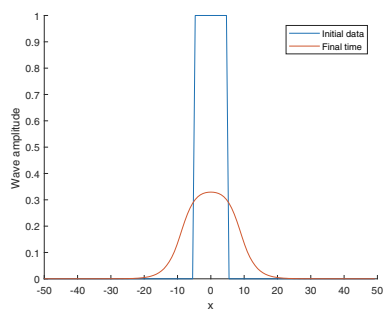

(c) $\psi_{0}$ and numerical solution at $T=2$.

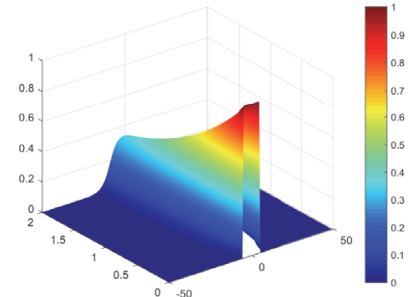

(d) Evolution of the numerical solution when $t \in(0,2)$.

Figure 5. Smoothing effect of the regularized Schrödinger equation. 
Moreover, we suggest to recall the idea of using a useful numerical schemes, whereby optimal order convergence is obtained under data of low regularity. For homogeneous parabolic PDE there are many contributions on convergence treating cases of non-smooth data, we refer to [11], [15]. In addition, for non-homogeneous parabolic PDE, We refer to the work of Khaliq and Wade [9] in which the authors used the smoothing Crank-Nicolson scheme (a Crank-Nicolson scheme modified by some backward Euler steps).

\section{Appendix : Z-Transformation}

One main tool of this work is the Z-transformation ([7] and Appendix B: Z-transformation in [5]) which is the discrete analogue of the Laplace-transformation. In this work we use it to construct the discrete-time resolvent and to prove the exponential stability of the semi-discrete problem.

Definition 3.1. (Z-Transform [7], [5]) The formal connection between a sequence and a complex function given by the correspondence

$$
Z\left\{u_{n}\right\}=\widehat{u}(z):=\sum_{n=0}^{+\infty} u_{n} z^{-n}, \quad z \in \mathbb{C}, \quad|z|>R_{\widehat{u}}
$$

is called Z-transformation. The function $\widehat{u}(z)$ is called Z-transformation of the sequence $\left\{u_{n}\right\}, n=$ $0,1, \ldots$ and $R_{\widehat{u}} \geq 0$ denotes the radius of convergence.

The discrete analogue of the Differentiation Theorem for the Laplace transformation is the shifting theorem:

Theorem 3.2. (Shifting Theorem [7]) If the sequence $\left\{u_{n}\right\}$ is exponentially bounded, i.e. there exist $M>0$ and $\gamma$ such that

$$
\left|u_{n}\right| \leq M e^{\gamma n}, \quad n=0,1, \ldots
$$

then the Z-transformation $\widehat{u}(z)$ is given by the Laurent series (40) and for the shifted sequence $\left\{v_{n}\right\}$ with $v_{n}=u_{n+1}$ holds

$$
Z\left\{u_{n+1}\right\}=z \widehat{u}(z)-z u_{0}
$$

The initial values enter into the transformation of the shifted sequence. As a useful consequence of the shifting theorem we have:

$$
Z\left\{u_{n+1} \pm u_{n}\right\}=(z \pm 1) \widehat{u}(z)-z u_{0}
$$

Finally, we present the inverse Z-transformation:

Theorem 3.3. (Inverse Z-transformation [7]). If $\left\{u_{n}\right\}$ is an exponentially bounded sequence and $\widehat{u}(z)$ the corresponding Z-transformation then the inverse Z-transformation is given by

$$
u_{n}=Z^{-1}\{\widehat{u}(z)\}=\frac{1}{2 \pi i} \oint_{C} \widehat{u}(z) z^{n-1} d z, \quad n=0,1, \ldots,
$$

where $C$ denotes a circle around the origin with sufficiently large radius. 
Acknowledgements - The present paper is an outgrowth of the first author's thesis under the guidance of the second author to him he is well-acknowledged. This work was partially financially supported by the "PHC Utique" program of the French Ministry of Foreign Affairs and Ministry of higher education and research and the Tunisian Ministry of higher education and scientific research in the CMCU project number $17 \mathrm{G} 1505$.

\section{References}

[1] L. Aloui. Smoothing effect for regularized schrödinger equation on bounded domains. Asymptotic Analysis, 59(34):179-193, 2008.

[2] L. Aloui and M. Khenissi. Stabilization of schrödinger equation in exterior domains. ESAIM: Control, Optimisation and Calculus of Variations, 13(3):570-579, 2007.

[3] L. Aloui, M. Khenissi, and L. Robbiano. The kato smoothing effect for regularized schrödinger equations in exterior domains. In Annales de l'Institut Henri Poincaré C, Analyse non linéaire, volume 34, pages 1759-1792. Elsevier, 2017.

[4] L. Aloui, M. Khenissi, and G. Vodev. Smoothing effect for the regularized schrödinger equation with non-controlled orbits. Communications in Partial Differential Equations, 38(2):265-275, 2013.

[5] X. Antoine, A. Arnold, C. Besse, M. Ehrhardt, and A. Schädle. A review of transparent and artificial boundary conditions techniques for linear and nonlinear schrödinger equations. Communications in Computational Physics, 4:729-796, 2008.

[6] C. Bardos, G. Lebeau, and J. Rauch. Un exemple d'utilisation des notions de propagation pour le controle et la stabilisation de problemes hyperboliques. pages 11-31, 1989.

[7] G. Doetsch. Anleitung zum praktischen Gebrauch der Laplace-Transformation und der Z-Transformation. R. Oldenburg Verlag München, 1967.

[8] K.-J. Engel and R. Nagel. One-parameter semigroups for linear evolution equations. In Semigroup forum, volume 63, pages 278-280. Springer, 2001.

[9] A. Q. M. Khaliq and B. A. Wade. On smoothing of the crank-nicolson scheme for nonhomogeneous parabolic problems. Journal of Computational Methods in Sciences and Engineering, 1(1):107-123, 2001.

[10] K. Liu. Locally distributed control and damping for the conservative systems. SIAM Journal on Control and Optimization, 35(5):1574-1590, 1997.

[11] M. Luskin, R. Rannacher, and W. Wendland. On the smoothing property of the crank-nicolson scheme. Applicable Analysis, 14(2):117-135, 1982.

[12] E. Machtyngier and E. Zuazua. Stabilization of the schrödinger equation. Portugaliae Mathematica, 51(2):243-256, 1994.

[13] I. Nouira and M. Khenissi. Smoothing effect and exponential stability of discrete-time schrödinger equation with fractional regularization term. To appear in International Journal of Computer Mathematics.

[14] J. Prüss. On the spectrum of $c_{0}$-semigroups. Transactions of the American Mathematical Society, 284(2):847-857, 1984.

[15] R. Rannacher. Finite element solution of diffusion problems with irregular data. Numerische Mathematik, 43(2):309_ $327,1984$.

[16] J. M. Sanz-Serna and C. Palencia. A general equivalence theorem in the theory of discretization methods. Mathematics of computation, 45(171):143-152, 1985.

[17] R. M. Young. An introduction to nonharmonic Fourier series. Academic press, 1981. 